\title{
Modelling and evaluation of pulsed and pulse phase thermography through application of composite and metallic case studies
}

\author{
R. C. Waugh*, J. M. Dulieu-Barton and S. Quinn
}

Faculty of Engineering and the Environment, University of Southampton, Highfield, Southampton SO17 1BJ

*rcw1n09@soton.ac.uk

\begin{abstract}
A transient thermal finite element model has been created of the pulsed thermography (PT) and pulse phase thermography (PPT) experimental procedure. The model has been experimentally validated through the application of four case studies of varying geometries and materials. Materials used include aluminium, carbon fibre reinforced plastic (CFRP) and adhesively bonded joints. The same four case studies have also formed a basis for comparison between three experimental techniques: PT, PPT and the more established ultrasonic (UT) c-scan.

Results show PPT to be advantageous over PT due to its deeper probing as it is less influenced by surface features. Whilst UT is able to reveal all the defects in these case studies, the time consuming nature of the process is a significant disadvantage compared to the full field thermography methods.

Overall, the model has achieved good correlation for the case studies considered and it was found that the main limiting factor of the PT model accuracy was knowledge of thermal material properties such as conductivity and specific heat. Where these properties were accurately known the model performed very well in comparison with experimental results. PPT modelling performed less well due to the method of processing the PT data which aims to emphasise small differences. Hence inaccuracies in inputted values such as material properties have a much greater influence on the modelled PPT data. The model enables a better understanding of PT and PPT and provides a means of establishing the experimental set-up parameters required for different components, allowing the experimental technique to be appropriately tailored to more complex situations including bonded joints or structures where several materials are present.
\end{abstract}

The paper ends with a section on defect detectability based on thermal diffusivity contrast between the defect and the bulk material. It shows that in aluminium, because 
of its higher conductivity, greater thermal contrast is achieved for small differences in diffusivity. Regions where the diffusivity ratio between defect and bulk materials was insufficient to provide thermal contrast for defect identification were found. PPT phase data is shown to reduce the extent of such regions increasing the detectability of defects. Effusivity is introduced as a means of determining the thermal contrast between the defect and non-defective areas and hence establishing the defect detectability.

\section{Introduction}

Adhesively bonded joints are often preferable to their mechanical counterparts due their ability to decrease the weight of the joint. Bonded joints enable a more even distribution of load transfer through the joint, avoiding the high stress concentrations typically associated with mechanical fastenings. The increased use of fibre reinforced composite materials, where machining the material for mechanical fastenings has the potential to initiate damage, has led to an increase in the use of adhesive joints in engineering structures. There is also an increasing trend towards the use of adhesively bonded composite patch repairs on damaged structures as a means of reinforcement [1]. Adhesive bonds are prone to several types of defect which have previously limited the range of application of such joints. To enable more widespread use of adhesive joints in structural applications all types of defects must be accurately and reliably detected. Defects typically found in adhesive joints may be categorised in three groups; voids, inclusions and kissing defects [2]. A void is the inclusion of air in the adhesive layer. Air may be introduced into the resin during the mixing of a two-part adhesive or during joint construction where a lack of contact occurs between the adhesive and adherend, resulting in an air gap in that area. Inclusions involve the physical inclusion of a foreign material of relatively large volume when compared to the bond line thickness. Kissing defects are a reduction of adhesion between the adhesive and adherend without affecting the thickness of the adhesive layer, hence there is no gap between the adhesive and the adherend. Such defects currently cannot be reliably and consistently detected [3,4].

Established NDE techniques typically used in industry for material assessment today include ultrasonic testing [5], radiography [6], shearography [7, 8] and thermography [9]. The drive to apply adhesive joints in a wider range of applications is increasing the demand for the development of new NDE techniques suitable for their efficient and effective assessment. Many current NDE methods are able to identify defects; however, 
no individual technique is able to reliably identify the reduced adhesion associated with kissing defects. Research has been carried out investigating advanced ultrasonic methods which has shown promise for identification of kissing defects [10, 11], however, this remains a point by point process and as such is not ideal for large scale applications. Thermographic techniques have the advantage of being full field, noncontact and fast. Previous studies have shown the potential use of thermography for identification of defects in adhesive bonds [12]. Most studies have focussed on specific applications or specific material or joint configurations. This work aims to develop a model that is able to simulate pulsed thermography (PT), and pulse phase thermography (PPT) through processing of the data, which can be tailored to specific components. As experimental parameters in PT and PPT change for different components, the use of such a model will allow experimental parameters to be tailored to the application without the need for extensive, time consuming and potentially expensive preliminary experiments. Finite element approaches have been used previously to model pulsed thermography. In [13] carbon-carbon composites with voids are considered and in [14] steel plates with flat bottom holes are considered by using a secondary heat source as the defect in the FEA. Both models are developed for a specific experimental set up and only a single test case is shown. The current work aims to expand upon this, presenting a series of case studies of various materials and configurations. Although kissing defects are not explicitly studied in the current work, the development of the modelling methodology enables the limits of defect identification to be focused on, in terms of their thermal properties. It should however be emphasised that if there is no thermal contrast between a defect and surrounding material then it cannot be identified by thermography alone; this is the subject of a future paper [15] where it is shown the application of a small load can help enhance thermal contrast and hence aid identification of kissing defects.

As a significant amount of research, e.g. [16], is being carried out into the use of ultrasonic techniques for detection of defects in composite materials, water coupled ultrasound (UT) c-scans of each defect have been included for comparison of different experimental techniques. While it is acknowledged that other forms of UT are more frequently used in the field, these images allow a representative comparison of UT and the thermographic techniques considered. 


\section{NDE Techniques}

Three NDE techniques have been studied for the detection of defects. Comparisons of experimental results are drawn between the three approaches of PT, PPT and UT.

\subsection{Pulsed and pulse phase thermography}

PT and PPT make use of highly sensitive infra-red detection systems and are carried out in either transmission or reflection mode. In transmission the heat source is on the opposite side of the component to the infra-red detector. The work described in the present paper focuses on reflection mode where the heat source and the detector are on the same side of the component. Thus reflection mode is a much more useful tool for industrial applications where only one side of a component or structure may be accessible.

In PT the surface of the component under investigation is exposed to a pulse of thermal energy. The thermal decay of the surface is then monitored as the heat conducts through the sample, see Fig. 1. If the material under the surface is uniform across the region of interest then the temperature of the front surface will decay uniformly. However, if the thermal front meets a region of different thermal properties, such as a subsurface defect, the surface temperature directly over the defect will be at a different temperature to the surrounding surface as the heat transfer has been perturbed in the region of the defect. For example, an air gap in aluminium has lower thermal conductivity than the aluminium and so the propagation of the thermal front is slowed in this area and is detected as a hotspot in the surface data during the thermal decay.

In reflection PT and PPT the surface of a component is monitored using an infra-red photon detector. A thermal pulse is impinged on the surface of the component by means of a heat source such as a flash lamp. A sequence of thermal images of the surface temperature is recorded for a period of time after the pulse. In PT the reduction in surface temperature across the surface of the component is studied. Any variation in the thermal decay rate across that surface is indicative of a variation of component configuration or sub-surface properties. 


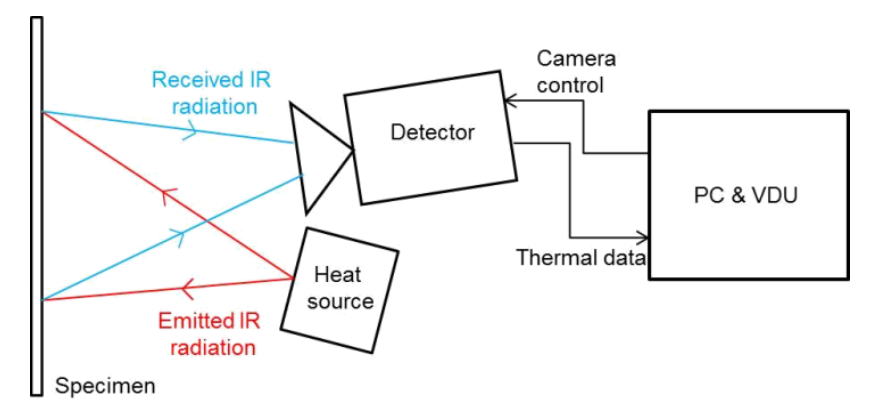

Fig. 1 Experimental apparatus for pulsed and pulse phase thermography showing heat pulse application and thermal decay data collection.

Using a pulse of heat means that the temperature rise of the component is limited to a few degrees above the initial temperature so damage due to excessive heating does not occur. The required duration of the pulse is dependent upon the thermal properties of the component and its geometry. For example, a thicker sample made of material with a lower thermal conductivity will require a longer thermal excitation [17]. The time delay between the application of the pulse and the appearance of the defect is related to the defect depth. Prediction of defect depth using thermography is not covered in this work but this is the area of research in several other papers, e.g. [18], that may later be incorporated into the model presented in this work.

Lock-in thermography (LT) or modulated thermography applies a continuous sinusoidal heat source of a particular frequency to the material surface. The exact time dependence of the output and input signals is monitored using a lock-in amplifier [19]. The response recorded is also sinusoidal, so the phase and magnitude of the output may be found via application of a fast Fourier transform (FFT). The phase data obtained from the FFT varies between defect and defect-free areas. Phase data is less affected by local surface optical variations and so is reported to probe approximately twice the depth of the temperature data [20]. A particular frequency heat pulse will probe a particular depth in the material governed by the materials heat transfer properties. The experiment must be repeated at a range of frequencies to probe the full thickness of the material or up to the maximum penetration depth for that material.

PPT uses the experimental procedure used in PT combined with the application of a FFT to calculate phase and magnitude values, as in LT. The start point of the FFT is a user defined datum taken as the frame after the peak of the flash. The peak flash frame is not used as the IR detector elements are typically saturated at this point. The phase values found using the FFT in PPT are associated with the change of signal relative to 
the datum using a range of frequencies as the reference. By using a heat pulse instead of a single frequency as used in LT multiple frequencies of heat are provided. These can be processed simultaneously hence revealing defects at a range of depths in a single test. As phase is not directly a temperature measurement, surface effects such as uneven heating are reduced, which enables deeper material inspections [19].

\subsection{Water coupled ultrasound-c-scan}

The current paper uses water coupled UT c-scans as a means of comparison for the results from the thermography methods, thereby providing a means of independent validation and for assessing the reliability of the thermographic identification of defects. The case studies of interest contained thin defects, which required a very high frequency transducer to be able to resolve the defect in the samples using the pulse echo method. An alternative method, known as the double through transmission method, allows a lower frequency transducer to be used with a back plate of a high density material placed behind the sample. The signals that have travelled through defective and defect free areas of the sample will have different amplitudes and so the signals received back, reflected from the back plate, will also vary in amplitude. These signals give no information about defect depth as they have travelled through the full thickness of the sample and so produce a shadow style image, showing only the lateral extent of the defect. Defect depth information is available for the pulse echo method where high frequency transducers were used and defect thicknesses can be resolved.

\section{Experimental procedure}

The IR detector used in this work is a Cedip Silver 480M photon detector with an indium antimonide ( $\mathrm{InSb}$ ) sensor array with a detectable wavelength range of $2-5.5 \mu \mathrm{m}$. The detector is cooled using a Stirling pump maintaining an operating temperature of 77 $\mathrm{K}$ and can achieve a frame rate of up to $383 \mathrm{~Hz}$ at the full detector array size of $256 \mathrm{x}$ 320. There are two heating sources used in the experimental work presented. Thinner samples with shallower defects have been tested using a Nikon Speedlight SB-600, which is a simple external camera flash unit that was triggered remotely. The second heat source was a small standard IR heat lamp. As the IR lamp did not turn on or off instantaneously a shutter was attached to the lamp to allow the heat exposure to be applied as a square pulse shape. The duration of exposure was controlled with the shutter. The assumption of a square pulse was deemed acceptable if, at the IR detector 
data collection frequency, an instantaneous increase and decrease of temperature was observed.

The general experimental set-up is illustrated in Fig. 1. A stand-off distance between the detector and sample was maintained at $250 \mathrm{~mm}$ creating a suitable sized region of interest and the distance between the sample and the heat source was maintained at 180 $\mathrm{mm}$ which provided uniform heating across the region of interest. The detector was aligned to be just off normal $\left(<10^{\circ}\right)$ to the sample to avoid reflections of the detector in the captured images and the flash was normal to the surface to enable uniform illumination across the surface. The heat source was located so the heat pulse was directed at the centre of the region of interest. The size of the region of interest was chosen so it was large enough to detect the defects under consideration and include their surrounding area but only required a single heat source for the thermal stimulation; a typical size was $100 \times 80 \mathrm{~mm}$.

The thermal data was processed into the frequency domain using a fast Fourier transform (FFT). Each pixel of the thermal images is converted into a thermal vector through time upon which the FFT is applied. As there is no lock-in signal for the FFT a frequency range and number of intervals within this range are selected to generate sampling frequency sine waves to carry out the FFT. The sampling frequency range and interval between sampling frequencies is defined by the user.

Water coupled UT c-scans were produced for each case study. As the defect locations and sizes were known the scanned area was tailored to the size of the defects. The first two case studies used the transmission method, which allowed use of a mid-range frequency transducer $(15 \mathrm{MHz})$ because it was not necessary to resolve features within the thickness of the sample. Case study 3 illustrates the application of a very high frequency $(50 \mathrm{MHz})$ transducer using the pulse echo method, which was able to resolve the different layers through the thickness of a lap joint of $2.0 \mathrm{~mm}$. It was necessary to be able to resolve the thin layers of CFRP and adhesive in case study 4 as they were adhered to a thick piece of concrete. Due to its structure, concrete is a highly attenuating material and as such UT signal with a high enough frequency to collect defect information would not be able to pass through it, so the double through thickness method was not an option. The $50 \mathrm{MHz}$ transducer was also used in this case. 


\subsection{Case studies}

Four case studies are studied, covering various materials and bonded/non-bonded configurations detailed in Table 1 with schematics of the samples shown in Fig. 2. Both experimental and modelled data is presented for all cases.

Table 1 Specimen information
\begin{tabular}{|c|c|c|c|c|c|}
\hline $\begin{array}{c}\text { Case } \\
\text { study }\end{array}$ & Description & Material & $\begin{array}{c}\text { Defect } \\
\text { material }\end{array}$ & Defect depth & $\begin{array}{c}\text { Full } \\
\text { sample } \\
\text { thickness }\end{array}$ \\
\hline 1 & $\begin{array}{c}\text { Aluminium with flat } \\
\text { bottom hole }\end{array}$ & $\begin{array}{c}\text { Al95 Dural } \\
\text { Aluminium alloy }\end{array}$ & Air & $0.3 \mathrm{~mm}$ & $1.5 \mathrm{~mm}$ \\
\hline 2 & CFRP & UD CFRP [UD] & $0.2 \mathrm{~mm}$ PTFE & $0.375 \mathrm{~mm}$ & $1.0 \mathrm{~mm}$ \\
\hline 3 & $\begin{array}{c}\text { CFRP-CFRP } \\
\text { adhesive lap }\end{array}$ & UD CFRP [0,90] & $0.2 \mathrm{~mm}$ PTFE & $0.7 \mathrm{~mm}$ & $2.0 \mathrm{~mm}$ \\
\hline 4 & $\begin{array}{c}\text { CFRP-Concrete } \\
\text { joint }\end{array}$ & UD CFRP [UD] & $0.2 \mathrm{~mm}$ PTFE & $1.75 \mathrm{~mm}$ & $11.75 \mathrm{~mm}$ \\
\hline
\end{tabular}
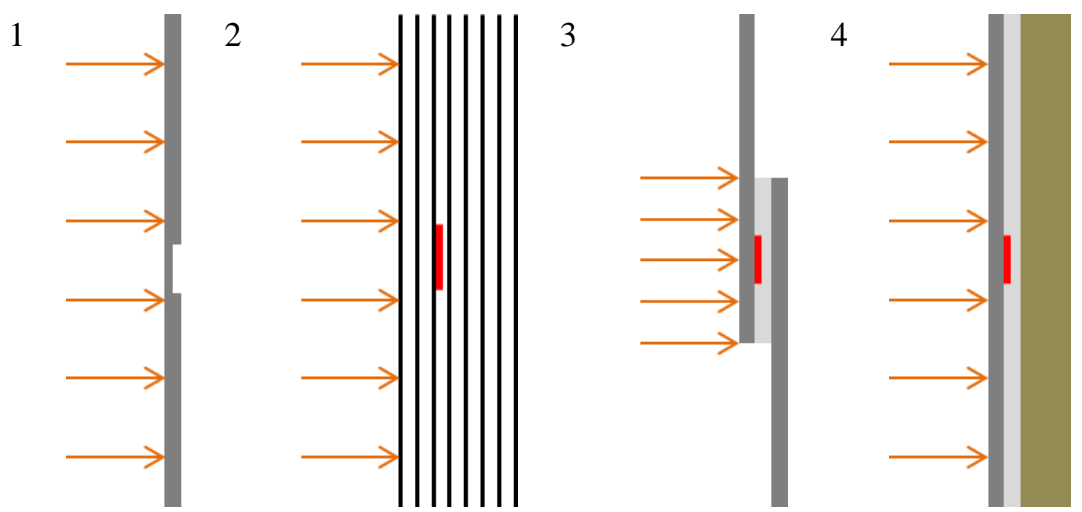

Fig. 2 Schematics of the four case study samples with heated surface indicated by orange arrows. 1) flat bottom hole milled into aluminium, 2) CFRP with PTFE inserted between plies 3 and 4 from the front, 3) a CFRP single lap joint made with epoxy adhesive with a PTFE insert in the bond line between front adherend and adhesive and 4) CFRP adhered to a concrete slab using epoxy adhesive.

\subsection{Experimental results}

For each case study three images are presented. The first is a frame from the thermal data collected after heating; a background subtraction of a frame prior to heating is carried out in order to observe the change in temperature after heating, as is used for analysis in PT. The second image is a phase image produced using PPT. The third image is a water coupled ultrasound image of the defect, which has been included for the comparison. The thermal PT and PPT phase images are taken from the same data set and so their observation window is the same. The ultrasound images have been taken independently and as such the observation windows vary from the thermography data. For quantitative analysis profile plots have been taken across the centre of defects 
along a horizontal line through the centre of the defect in the PT and PPT images. Average values for the readings from the non-defect area were obtained and subtracted from the data in the profile plots to show the contrast between defect and non-defect regions.

\subsubsection{CS1: Aluminium with flat bottom hole}

A $10 \mathrm{~mm}$ flat bottom hole was milled into the rear of a aluminium plate. The front surface of the aluminium sample was painted black to increase and standardise the emissivity and reduce reflections which are high for such a polished metallic sample.

All three of the experimental techniques presented here are able to clearly identify the simulated defect for CS1, Fig. 3. The thermal PT data, Fig. 3a, clearly reveals the circular defect although some surface features are also shown which appear to be scratches on the metal surface. Whilst in this particular case these surface features are small compared to the defect they are still visible and have the potential to lead to inaccurate identification of defects. The PPT phase image in Fig. $3 \mathrm{~b}$ appears to be of lower quality than the PT thermal image due to the processing of the results. It is apparent in Fig. 3b that the PPT data suffers from higher levels of noise degradation which is due to the data processing algorithm that derives the phase values (this is discussed further in section 4.3. [19]). Despite this the defect is still clearly identifiable. The phase data removes the surface features seen in the PT image which will allow clearer identification of defects. Fig. 4 shows the profile plots across the defect centre of both the PT and PPT with the defect clearly apparent. The $\Delta \mathrm{T}$ obtained for the defect is approximately $0.20 \mathrm{~K}$ and the $\Delta \phi$ obtained is $5^{\circ}$ using the flash light heat source. The UT data, see Fig. 3c, clearly shows the defect. The edges of the defect in this case are particularly clear as more of the UT signal is scattered at vertical boundaries, such as the edge of the milled hole, leading to the ring like appearance.

a) PT

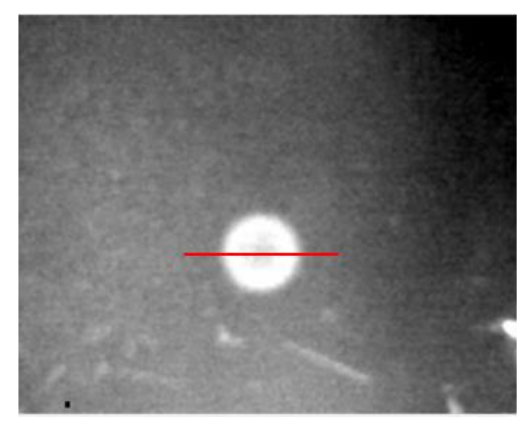

b) PPT

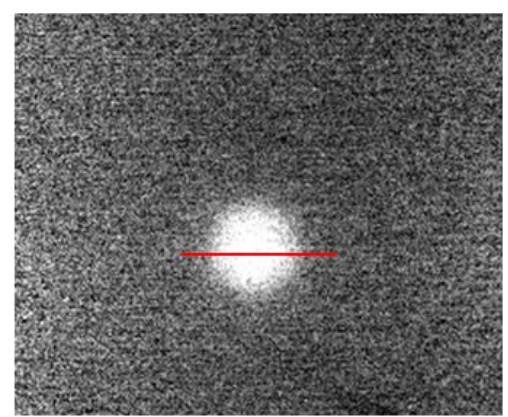

c) UT

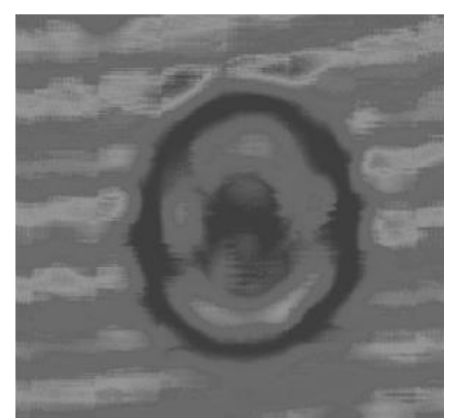


Fig. 3 Experimental data for CS1 recorded at $100 \mathrm{~Hz}$ : a) PT thermal image at $0.01 \mathrm{~s}$, b) PPT phase image at $0.19 \mathrm{~Hz}$ and c) UT image.

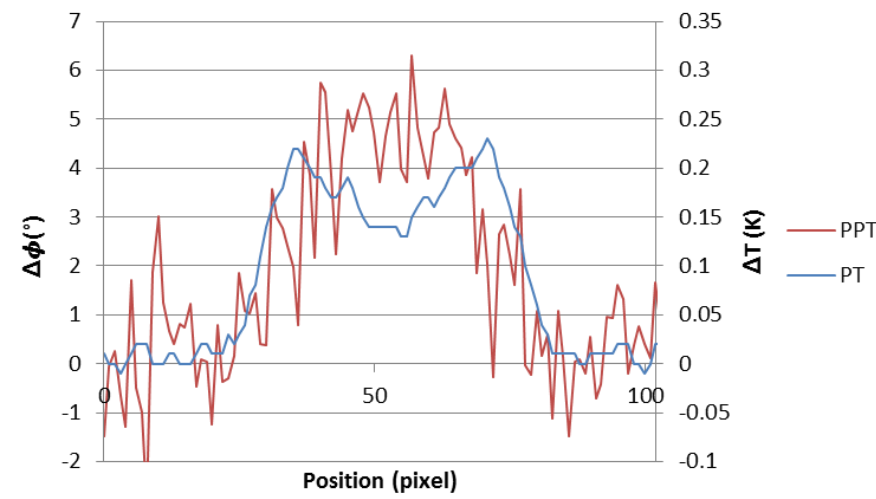

Fig. 4 Profile plots taken across the width of the defect visible in the PT and PPT data shown in Figure 3a \&b.

\subsubsection{CS2: CFRP}

The CFRP sample had an 8 ply unidirectional layup with a $10 \mathrm{~mm}$ circular PTFE defect inserted between plies 3 and 4 during manufacture giving a defect depth of $0.375 \mathrm{~mm}$.

Both the thermal PT and PPT phase data are able to clearly reveal the PTFE defect in the CFRP, see Fig. 5a and b. Fig. 6 shows profile plots taken across the defect in the PT and the PPT data. The thermal contrast obtained is approximately $0.04 \mathrm{~K}$ and the phase contrast is approximately $17^{\circ}$ across the defect. The profile shapes in the phase and thermal data are similar for this easily detectable defect. Although there is significant noise in both data sets, the periodic nature of the noise in the PT data is as a result of the surface peel ply imprint which is an artefact of the manufacturing process. The PPT cleans the noise from the surface but processing noise is introduced in the PPT for the reasons given in the previous case study. The defect revealed in the UT data is not as clear as the thermal methods; this may be due to the frequency of probe used being slightly lower than ideal. An improved image is possible; however, this demonstrates that a single UT scan set-up is not optimum for every sample. Additional probes are generally costly compared to tailoring the thermographic heat source to an application.

a) PT

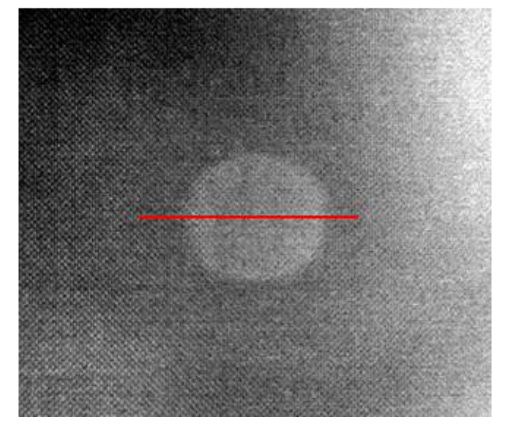

b) PPT

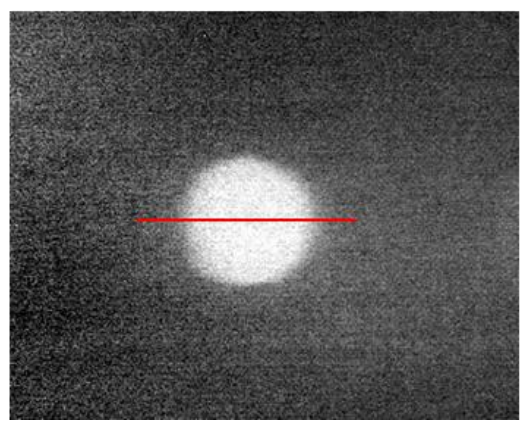

c) UT

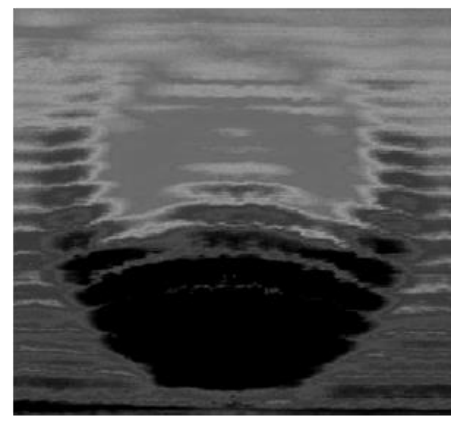


Fig. 5 Experimental data for sample 2: a) PT thermal image at $0.24 \mathrm{~s}$, b) PPT phase image at $0.175 \mathrm{~Hz}$ and c) UT image.

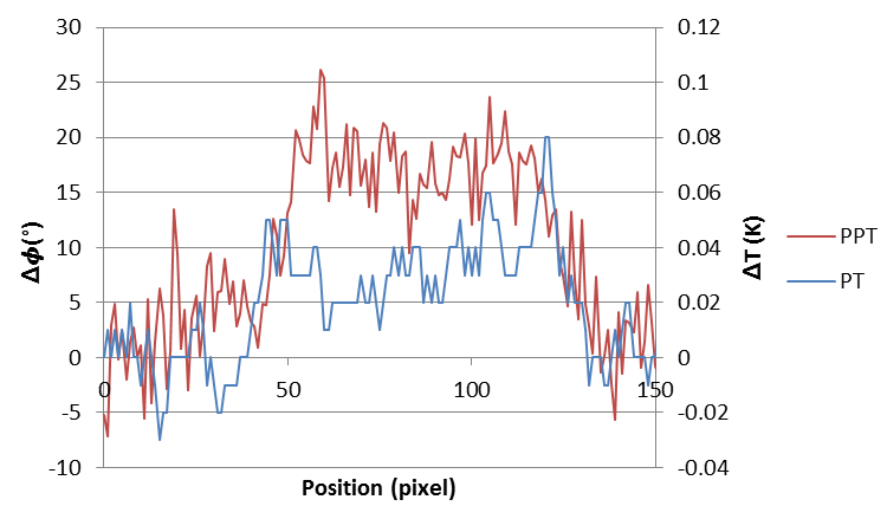

Fig. 6 Profile plots taken across the width of the defect visible in the PT and PPT data shown in Figure 4a \&b.

\subsubsection{CS3: CFRP-CFRP adhesive lap}

The third case study presented is a CFRP-CFRP adhesively bonded lap joint made using Araldite Rapid epoxy adhesive. A $10 \mathrm{~mm}$ square PTFE insert style defect was added to the bond line. Each piece of CFRP is a 4 ply panel with a layup of $[0,90]_{\mathrm{s}}$ resulting in a defect depth of $0.7 \mathrm{~mm}$.

The thermal PT data for CS3, Fig. 7a, does not clearly reveal the PTFE defect in the lap joint, however, when this data is processed the defect becomes visible in the PPT phase data, see Fig.7b. Whilst defects that were detected using PT were also detected by PPT, the reverse is not true, as is demonstrated in this case. The UT data for this sample, Fig. 7c, was taken using a very high frequency probe of $50 \mathrm{MHz}$ to allow the very thin features in the adhesive layer to be resolved. This results in a very clear image with a lot of detail but is a time consuming process taking approximately 15 minutes for this $20 \times 20 \mathrm{~mm}$ scan and this detail is not required for the identification of the defect. PPT was significantly quicker and simpler to carry out and gives sufficient information for the extent and position of the defect to be identified. The relative visibility of the defect in PT and PPT data is highlighted in the profile data shown in Fig. 8. As the thermal contrast is low, the PT $\Delta \mathrm{T}$ profile does not clearly identify a defect within the joint. However, the PPT $\Delta \phi$ data is able to indicate the presence of the defect. From CS1 and CS2 it is clear that when the defect is clearly visible in the PT thermal data the advantages of processing the data into PPT data are not apparent, however, from CS3 
where the defect is not visible in the PT data the usefulness of the phase data becomes apparent as the defect becomes identifiable.

a) PT

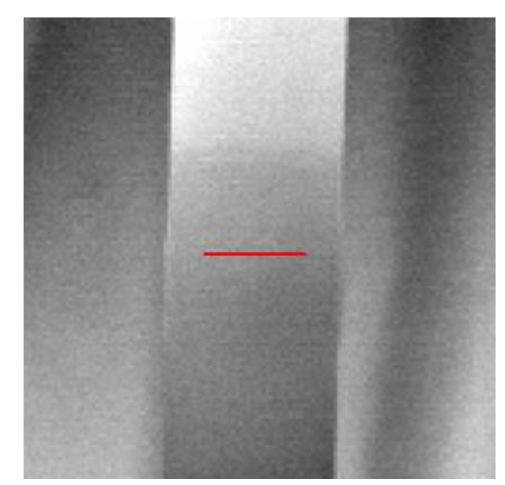

b) PPT

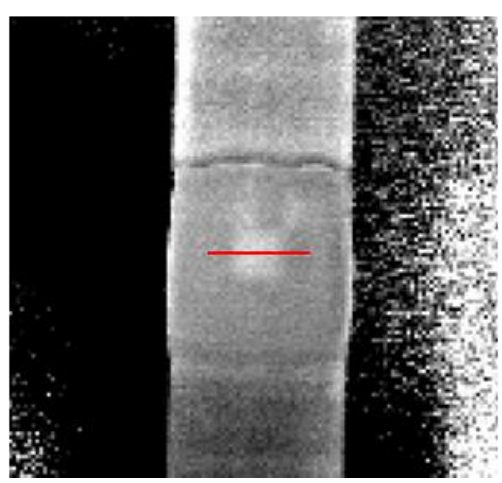

c) UT

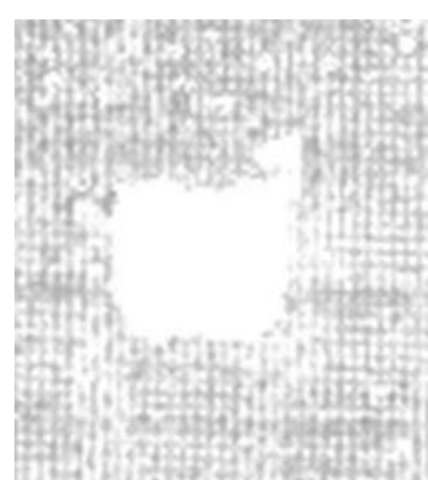

Fig. 7 Experimental data for sample 3: a) PT thermal image at $0.514 \mathrm{~s}$, b) PPT phase image at $0.053 \mathrm{~Hz}$ and c) UT image.

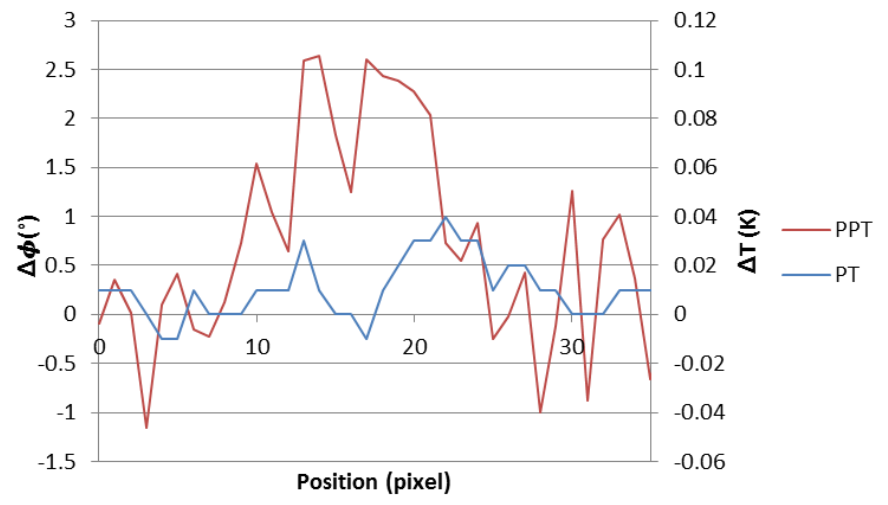

Fig. 8 Profile plots taken across the width of the defect visible in the PPT data shown in Figure 7a \&b.

\subsubsection{CS4: CFRP-Concrete joint}

Finally a case study is presented for an epoxy adhesive joint between a unidirectional 1 $\mathrm{mm}$ thick strengthening CFRP panel and concrete. PTFE inserts were added during the construction of the joint between two layers of adhesive, which was applied to each component to make the joint. The adhesive in this case is approximately $1.5 \mathrm{~mm}$ thick, adhering to industry standards for the application of CFRP patches for concrete repairs. This construction led to a defect depth of approximately $1.75 \mathrm{~mm}$.

The experimental data is presented in Fig. 9. The PTFE defect of interest is the crescent shape found to the right of both the thermal PT and PPT phase images, see Fig. 9a and b. The PPT phase image has removed the surface effects and reflections that are present in the PT image which allows clearer identification of defects. The UT image, Fig. 9c, clearly shows the PTFE defect as well as showing variations in the adhesive. Whilst in 
this case the defect remains clear amongst the adhesive variations, when smaller defects were studied they were found to be harder to distinguish from this adhesive information. This was not the case for PPT phase data.

$\Delta \mathrm{T}$ and $\Delta \phi$ profile plots are presented in Fig. 10. The thermal contrast between defect and non-defect is $0.4 \mathrm{~K}$ and the phase contrast across the defect is approximately $40^{\circ}$. The shape of the profiles across the defect in CS4 is quite different to CS1 and CS2 where profiles are found to be much squarer. This square profile is common for shallow defects where the defect is visible soon after the pulse has been generated. In the current case the defect was much deeper than the previous case studies at $1.75 \mathrm{~mm}$ deep so the maximum contrast PT image occurs after more time has passed. This additional time allows for an increased amount of lateral diffusion of the heat while the maximum thermal contrast is found over the centre of the defect hence creating a more sloped profile.

a) PT

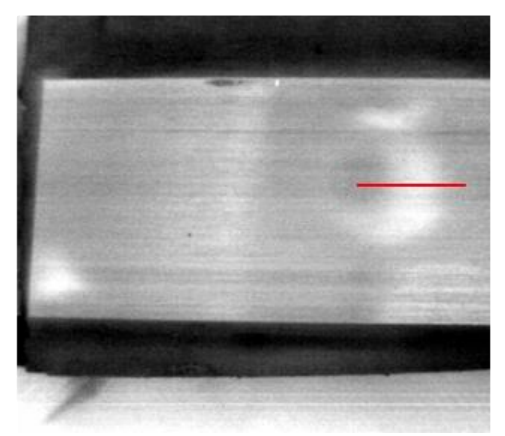

b) PPT

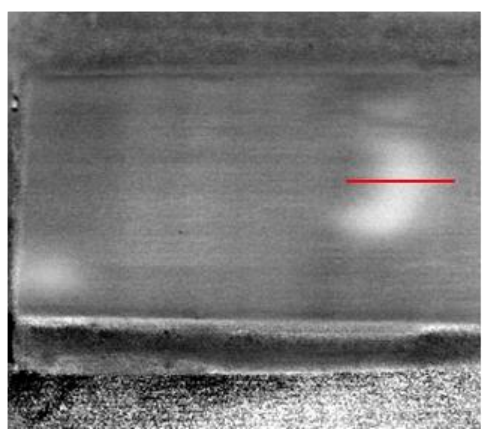

c) UT

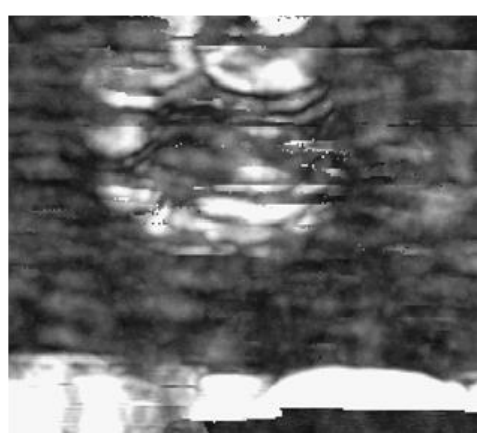

Fig. 9 Experimental data for sample 4: a) PT thermal image at $12.5 \mathrm{~s}$, b) PPT phase image at $0.84 \mathrm{~Hz}$ and c) UT image of major defect visible as a crescent shape in the PT and PPT.

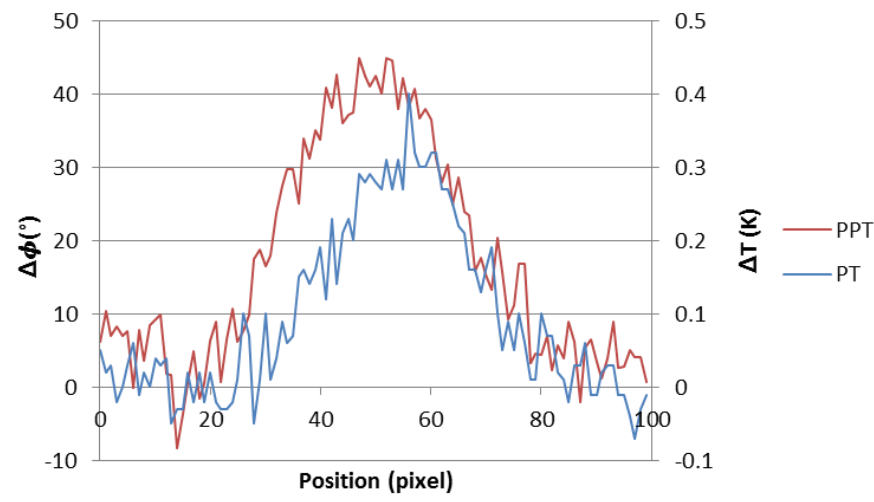

Fig. 10 Profile plots taken across the width of the defect visible in the PT and PPT data shown in Figure 9a \&b. 


\section{Finite element modelling of PT and PPT}

As the experimental procedures for PT and PPT are identical this model aims to recreate the experimental conditions and parameters used for both. The results obtained from the model are then compared to the PT results in each case. As the PPT phase results come from the processing of the PT data, the reliable recreation of the PT results is paramount and is therefore the focus of this work.

The aim of the model is to aid the physical understanding of PT/PPT and to allow experimental procedures to be defined for various materials or components. A 2D model has been developed as the advantages of a 3D model at this stage are not considered to outweigh the extra computational expense. Each sample is modelled as a cross section through the area where a defect exists, see Fig. 11. Animated results are produced that enable visualisation of the thermal front propagation through the sample whilst giving the comparative results of surface temperature values over defect and nondefect areas. The surface temperature values are equivalent to the PT data, which may then be processed using a FFT to obtain the phase values equivalent to PPT data.

The model has been developed using Ansys 12.1. The element type used is Plane55 which is a 4 node 2-D thermal solid element. The mesh size chosen was $0.2 \mathrm{~mm}$ which was kept consistent for all models developed in this work. The results generated using this mesh size were found to be convergent with the results given by smaller meshes so there was no loss of accuracy by selecting such a mesh size. The physics of the model is based on the fundamental heat transfer equation

$$
\frac{\partial T}{\partial t}=\gamma\left(\frac{\partial^{2} T}{\partial x^{2}}+\frac{\partial^{2} T}{\partial y^{2}}\right)
$$

where the thermal diffusivity, $\gamma$, is given as,

$$
\gamma=\frac{k}{\rho C}
$$

Parameters such as specific heat, $C$, density, $\rho$ and thermal conductivity, $k$, for each material are inputted into the model. For orthotropic materials thermal conductivity values parallel, $k_{\|}$, and perpendicular, $k_{-}$, to fibres are included in equation (2) which becomes

$$
\gamma=\frac{1}{\rho C}\left(k_{\|}+k_{\perp}\right)
$$




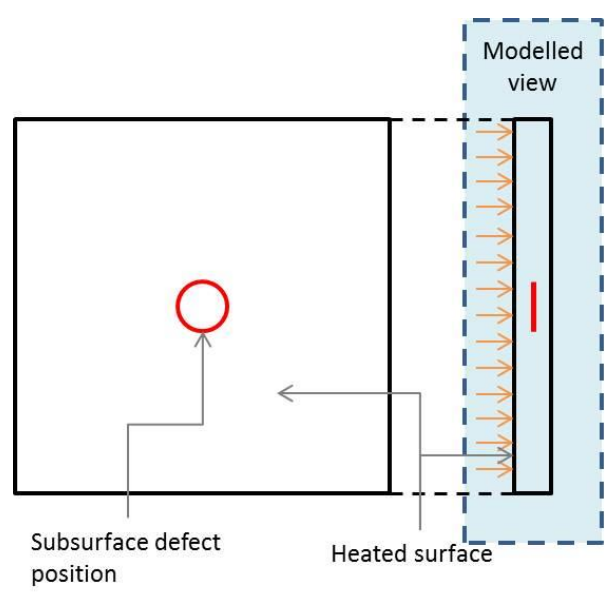

Fig. 11 Modelled view of example sample with defect in 2D model.

The modelled and experimental PT thermal data was then processed into PPT phase data, which has been carried out using a routine developed in Matlab, discussed in Section 4.3.

\subsection{Constructing the model}

When considering modelling PT there are several heat transfer factors to be considered and included. Firstly, the heat being added to the component as the pulse incident on the surface must be of realistic strength and duration. Secondly, the conduction of the heat through the material and the transfer of the heat across material boundaries must be considered. Finally, the interaction of the sample with the surrounding environment must be included. Convection of the heat away from the surfaces of the component must be possible in a realistic manner.

The parameters required to establish such a model include the conductivity, specific heat and density of each material. The surface heat transfer coefficients for any material open to the surrounding air must also be known. Experimental parameters must also be added, such as the ambient air temperature, pulse duration and strength, and data collection/sampling rate.

The initial condition of the model assumed the sample and surroundings were in thermal equilibrium. All free surfaces of the sample were set to be able to convect using the appropriate heat transfer coefficients found in the literature [19, 21]. The pulse was modelled by applying a uniform temperature across the front surface of the sample. The temperature value was determined using IR detector data from the experiments. This elevated temperature was then removed and the component allowed to return to ambient temperature. The model was processed in three load steps to simulate PT. Load step 
one was the initial condition with the sample and surroundings in equilibrium. Load step two was taken for the duration of the thermal pulse. The third load step was taken from the end of the pulse for a duration appropriate to the component, which captures the thermal decay of the component back to ambient temperature. The duration of each load step was varied as necessary. The interval between recorded data points was selected to be appropriate to capture the details of the heat transfer in that component. This recorded data interval is analogous to the IR detector recording frequency. The increase of the sampling frequency results in a more computationally expensive model, just as the increase of recording frequency would result in a very data intensive experiment. The minimum recording / sampling frequency should be used while still being able to capture the details of the thermal decay of a component.

\subsubsection{Assumptions / limitations}

There are some assumptions and limitations to the current model. The first restriction in its use is that knowledge of several material properties is required. This knowledge of properties includes those of the defect, hence identification of the defect material is necessary, which is not always practical. In this situation the model could be used to find the limiting values of properties of a defect material that would allow it to be detectable. Another assumption of the model is that there is perfect contact conductance between the surfaces of different materials within the component. As bonded joints are the main area of interest, where contact is assumed to be $100 \%$ for a good bond, then this assumption is considered reasonable. Finally, the model applies a uniform illumination across the surface of the samples. However, in experiments, while this is the ideal case there may be a thermal gradient on the surface that may lead to discrepancies in results between the model and experimental work, particularly towards the edges of observed areas. This thermal gradient should be considered when processing experimental results and efforts should be made to remove this error.

There are some more minor effects that have not been included in the model that are present in the experimental data. For all case studies the experimental peak pulse temperature recorded is consistently higher than that modelled. This exaggerated peak in the experimental data is caused by the reflection from the sample as the thermal pulse is incident on the surface; this is not replicated in the model. As this was acknowledged before the model pulse temperature was informed, the change in temperature caused by 
the heating pulse was taken from the difference in surface temperature before and immediately after heating to provide a model thermal pulse value that represented the actual change of temperature of the component surface.

\subsection{Finite element modelling results}

The modelled and experimental data comparison is based on three key points on the surface. The first point is taken as the surface data directly above the defect centre (DC). The second point is taken from towards the defect edge (DE) while still being over the defect. The final point is taken from a non-defect (ND) area away from the edge of the defect and the edge of the sample, this point is assumed to be unaffected by any lateral conduction. The ND data is taken at the same distance from the defect in both the experimental and modelled data. A schematic of the data point locations is given in Fig. 12 , and the equivalent data was taken from both modelled and experimental results.

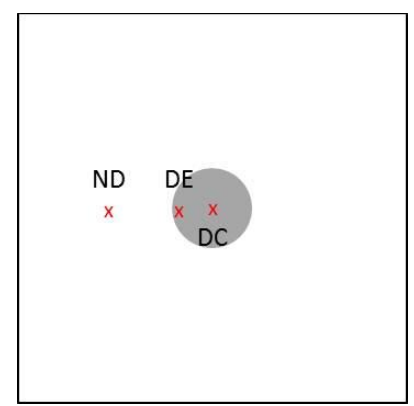

Fig. 12 Data point positions for comparisons between modelled and experimental data.

The $\Delta \mathrm{T}$ thermal decay data is presented initially to allow comparison of the modelled and experimental data, this data is presented as $\Delta \mathrm{T}$ data as a background subtraction of ambient temperature has been removed to observe the change in temperature on the surface over time caused by the external heating. A thermal contrast plot is also produced measuring the $\Delta \mathrm{T}$ between the defect and non-defect areas over time. This plot allows the time at which a defect is most clearly visible to be identified which may be used to inform experiments of the duration that a surface needs to be monitored after the pulse for maximum contrast between surface temperatures over defect and nondefect regions to be obtained.

\subsubsection{CS1: Aluminium with flat bottom hole}

A comparison of modelled and experimental data of CS1 is shown in Fig. 13a-d, where the trends present in the modelled data match those found in the experimental data for 
the three points considered. The initial decay section, prior to $1.5 \mathrm{~s}$, shows the surface temperature at ND decaying fastest after the pulse. This occurs because the component is thicker here and so the heat can be conducted through the thickness of the aluminium for longer, decreasing the surface temperature by a larger amount. At DC the aluminium is much thinner so the propagation of the thermal front through the material via conduction is stopped in the through thickness direction. The elevated temperature in that region is dissipated by either lateral conduction or convection from the surfaces. In the DC position there is less opportunity for lateral conduction as it is in the centre of the 'hot spot'. More lateral conduction occurs at the edge of the defect allowing DE to cool faster than DC. As convection becomes more dominant, after $1.5 \mathrm{~s}$, there is an inversion in the relative temperatures of the three points in the model. Where there is less material in location DC, a faster decrease in temperature is observed than where the material is thicker in location ND. This is caused by the ratio of surface area to volume of material being decreased for the thicker region. This effect is only visible in the model although could be present in the experimental data but as it is a relatively small variation in the thermal decay it cannot be distinguished in the experimental noise. The modelled and experimental PT data correlate within $\pm 0.03 \mathrm{~K}$.

The thermal contrast between the defect centre and non-defect areas is shown in Fig. 14. Due to the shallow depth of the defect and the high thermal conductivity of aluminium the peak in thermal contrast $\left(\Delta \mathrm{T}_{\text {peak }}\right)$ occurs very soon after the heating stimulus at $0.2 \mathrm{~s}$. The timing of this peak is predicted well by the model. The $\Delta \mathrm{T}_{\text {peak }}$ value is underestimated by the model at $0.06 \mathrm{~K}$ as opposed to the experimental value of approximately $0.09 \mathrm{~K}$. The discrepancy between modelled and experimental data is possibly caused by differences between the literature values of the material thermal properties and the true values of the sample used.

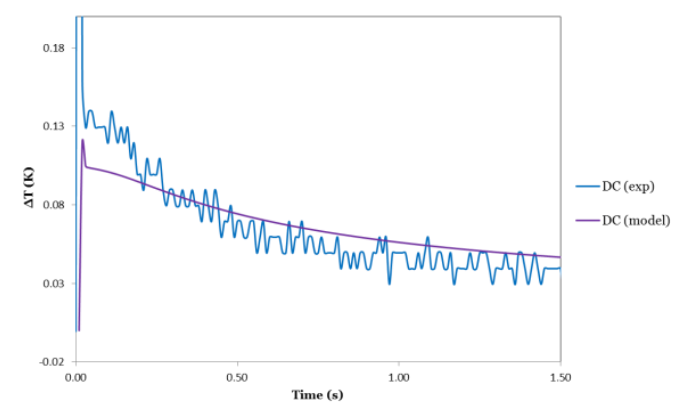

b

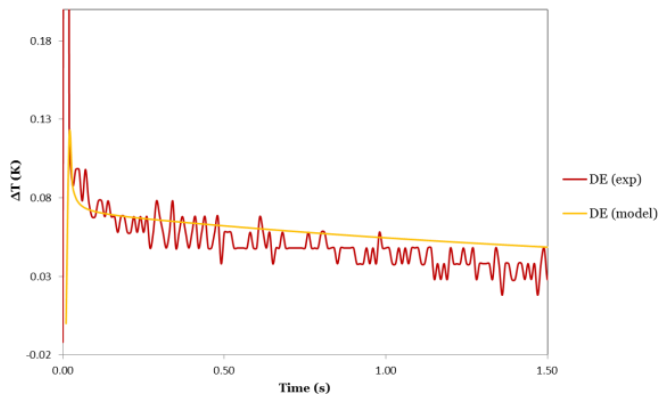


$\mathrm{c}$

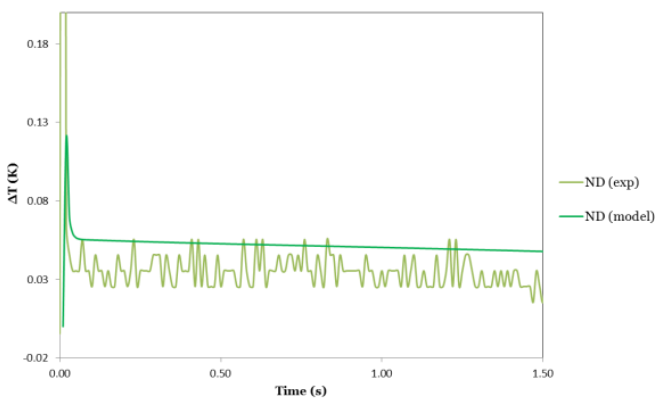

d

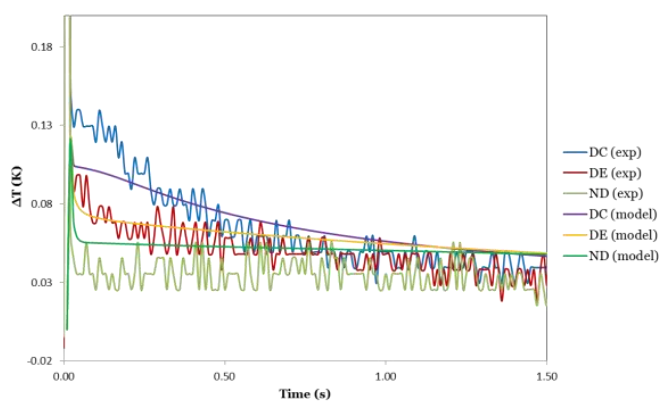

Fig. 13 Surface temperature data comparison of PT data for CS1 showing modelled and experimental data for a) DC, b) DE, c) ND and d) all three data points.

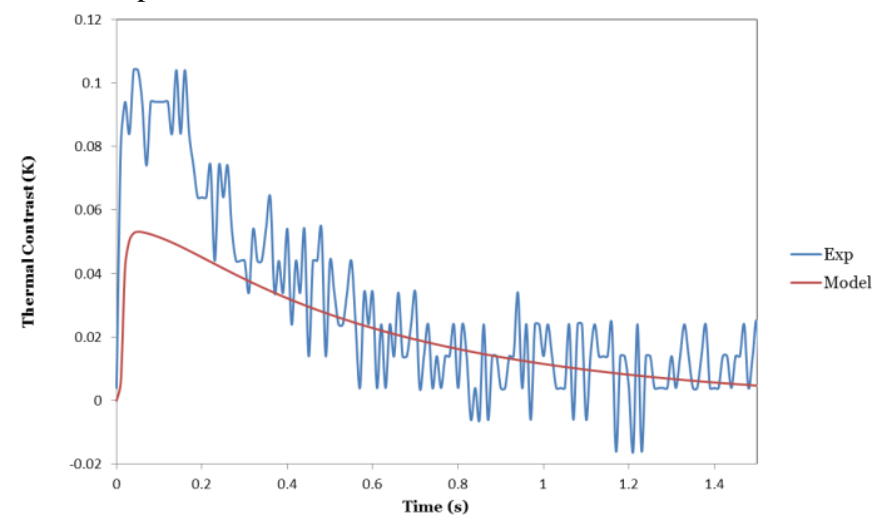

Fig. 14 Modelled and experimental surface thermal contrast data between defect and non-defect regions

\subsubsection{CS2: CFRP}

For CS2 the model data is in good agreement with the experimental PT results within \pm $0.04 \mathrm{~K}$, see Fig. 15a-d. The DC position is slowest to decay as the thermal front meets the PTFE defect as it propagates in this area. PTFE has a lower thermal conductivity than the surrounding CFRP. The DC stays at a higher temperature than the DE due to the increased limitations of lateral conduction, as previously discussed for CS1. The inversion of decay rates seen in CS1 is not present as the total component thickness is consistent, so cooling by convection will be more uniform than in the previous example as the ratio of volume of material to surface area is continuous across the sample.

Fig. 16 shows thermal contrast between defect and non-defect areas for the modelled and experimental results. The time of $\Delta \mathrm{T}_{\text {peak }}$ in the model occurs at $0.2 \mathrm{~s}$ whereas in experiments it occurs slightly earlier at approximately $0.13 \mathrm{~s}$. The decay of thermal contrast between DC and ND is faster in the model than in the experiment which may be attributed to the literature based material properties entered in the model. 
a

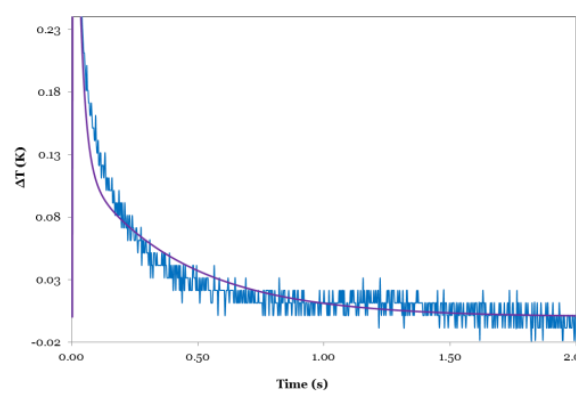

$\mathrm{c}$

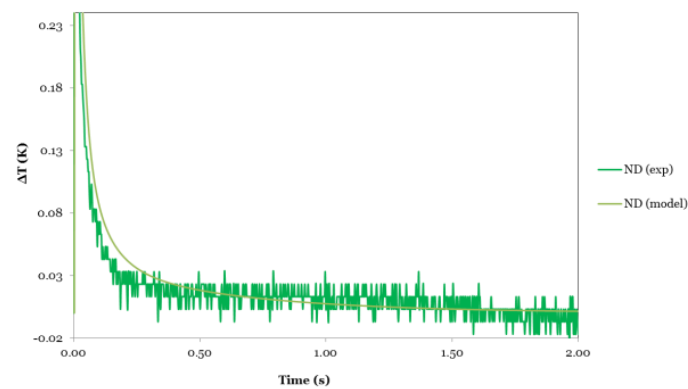

$\mathrm{b}$

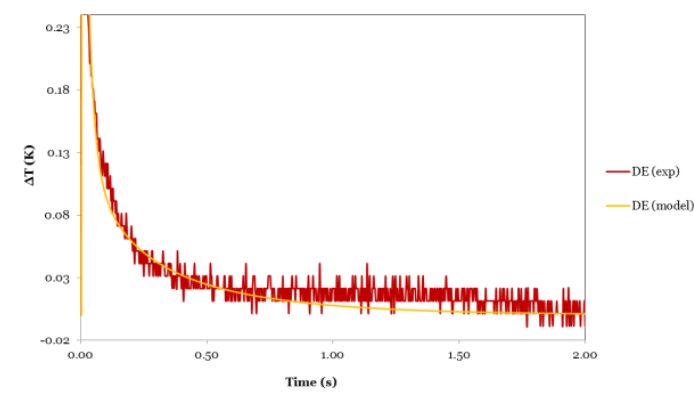

d

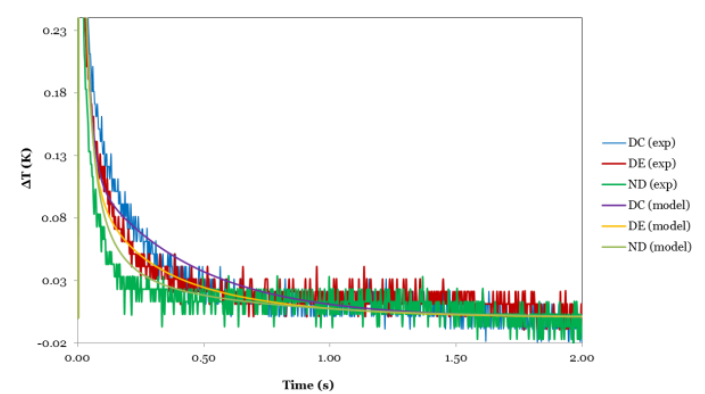

Fig. 15 Surface temperature data comparison of PT data for CS2 showing modelled and experimental data for a) DC, b) DE, c) ND and d) all three data points.

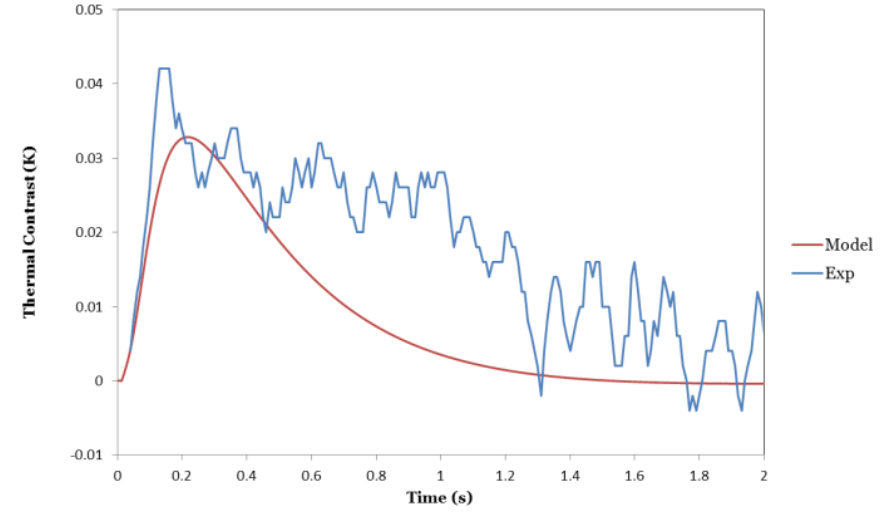

Fig. 16 Modelled and experimental surface thermal contrast data between defect and non-defect regions

\subsubsection{CS3: CFRP-CFRP adhesive lap joint}

The modelled data for CS3 is also in good agreement with the experimental results within $\pm 0.05 \mathrm{~K}$, Fig. 17a-d. The error has slightly increased as the component geometry has increased in complexity, however, a strong correlation remains.

Where very low thermal contrast is obtained as for the lap joint sample the experimental noise becomes significant. The profile plot in Fig. 18 shows that the magnitude of $\Delta \mathrm{T}_{\text {peak }}$ is accurately predicted at $0.03 \mathrm{~K}$ however the peak occurs later at $0.3 \mathrm{~s}$ in the model compared to approximately $0.2 \mathrm{~s}$ in the experimental data. 
a

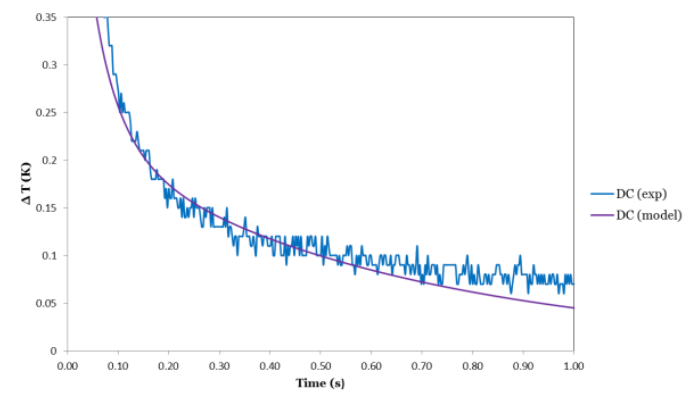

C

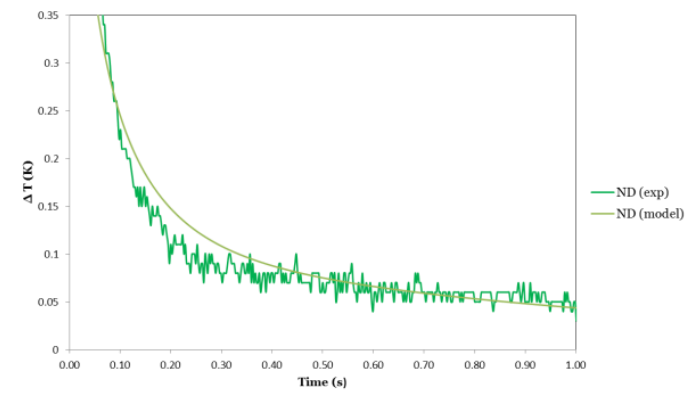

$\mathrm{b}$

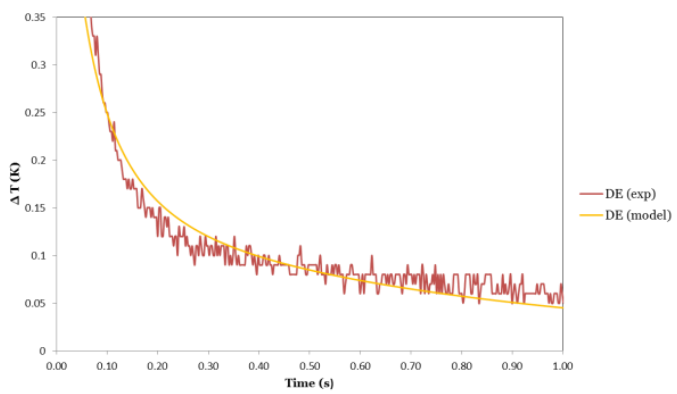

d

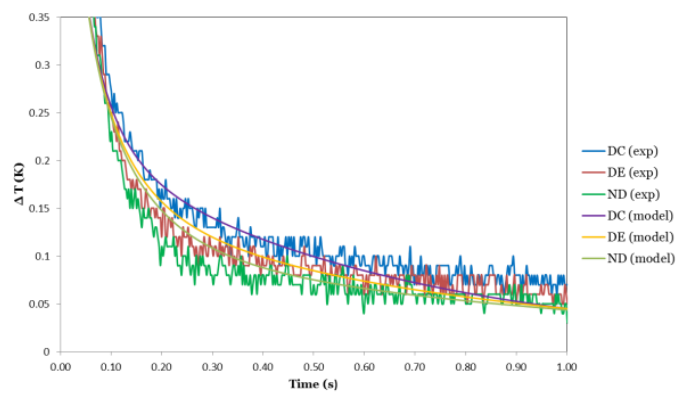

Fig. 17 Surface temperature data comparison of PT data for CS3 showing modelled and experimental data for a) DC, b) DE, c) ND and d) all three data points.

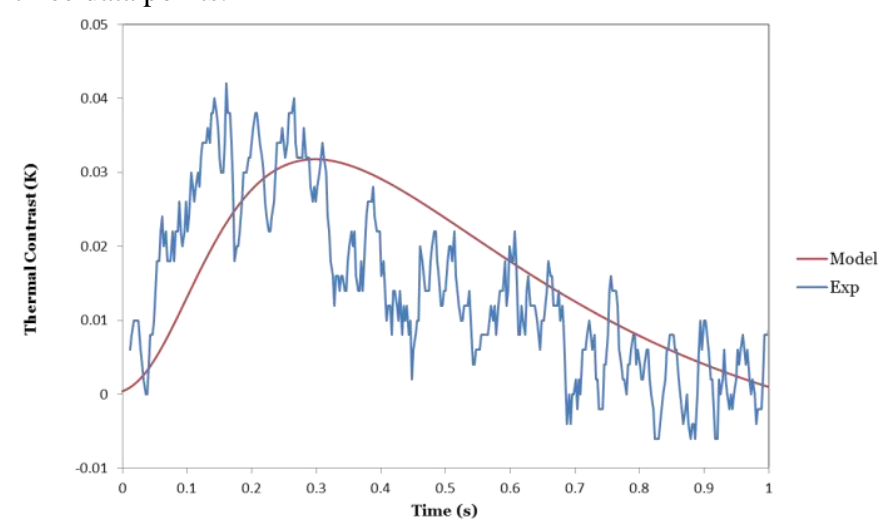

Fig. 18 Modelled and experimental surface thermal contrast data between defect and non-defect regions

\subsubsection{CS4: CFRP-Concrete joint}

In CS4 there is a discrepancy between the modelled and experimental data, as the decay of the model surface temperature is faster than that in the experiment, see Fig. 19a-d. The range in temperatures between the three points is also reduced to only approximately $0.07 \mathrm{~K}$ for the modelled data at $20 \mathrm{~s}$ compared to $0.3 \mathrm{~K}$ for the PT thermal data. It is believed that this is likely to be caused by inaccuracies in the material properties in the model. For less common materials, such as the adhesive used in this case as the sample was not manufactured by the authors, exact material properties were not available and so estimated values taken from literature were used, however this is reflected in the accuracy of the model. The CS4 model data decay in the same manner 
as the experimental data with the DC staying at an elevated temperature for longest and the ND cooling fastest although the temperature range between them is reduced. The model has a weaker correlation with the experimental data of $\pm 0.5 \mathrm{~K}$.

The thermal contrast data presented in Fig. 20 highlights the discrepancies between the model and experimental data caused by the inaccuracies in the thermal properties used in the model. The magnitude of $\Delta \mathrm{T}_{\text {peak }}$ is slightly over predicted by the model at $0.29 \mathrm{~K}$ compared to $0.24 \mathrm{~K}$ however the shape of the plot is quite different. It appears that the heat transfer within the model material is much faster than in the real materials leading to this much sharper peak. These case studies demonstrate that when the exact material is known a good estimate of material properties may be obtained from the literature however when materials are unknown, as in CS4 which was manufactured by a third party, estimated properties will cause discrepancies between modelled and experimental results.

a

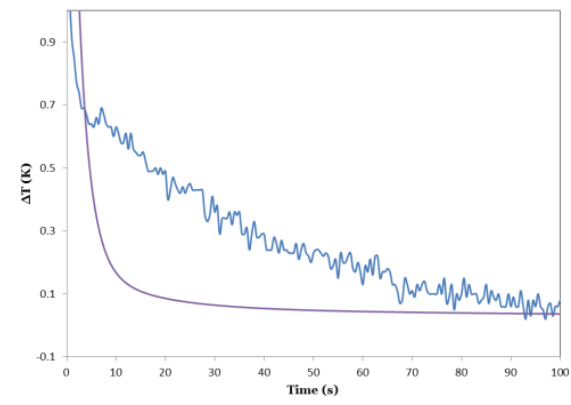

$\mathrm{c}$

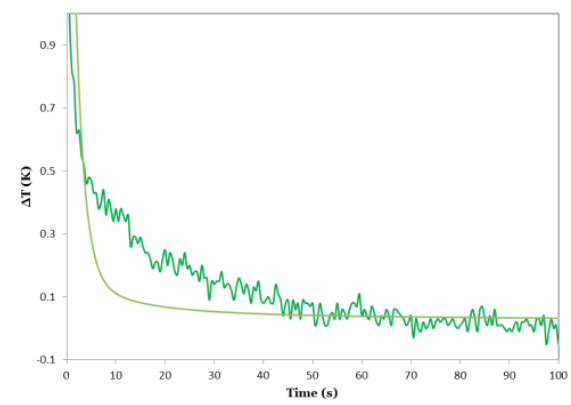

b

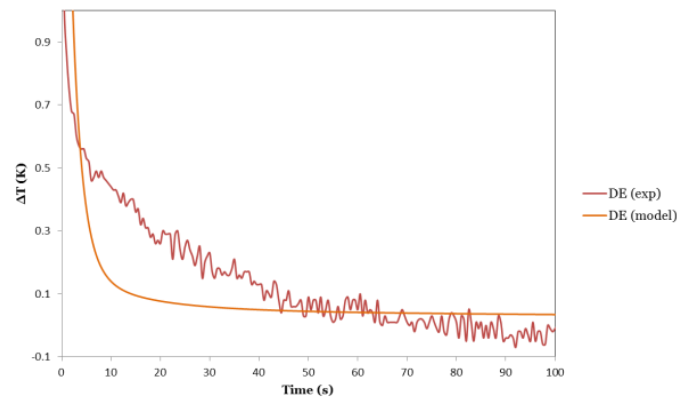

d

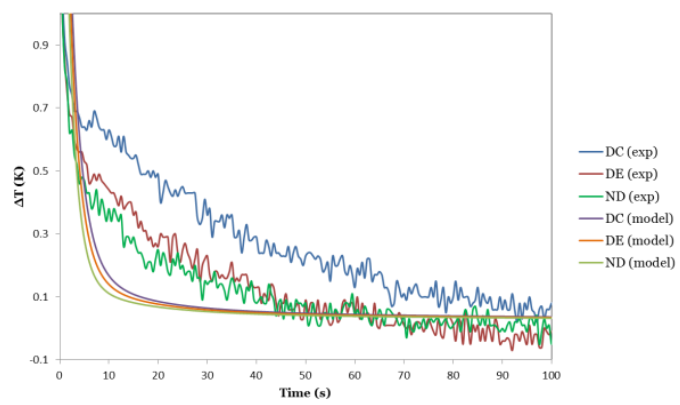

Fig. 19 Surface temperature data comparison of PT data for CS4 showing modelled and experimental data for a) DC, b) DE, c) ND and d) all three data points. 


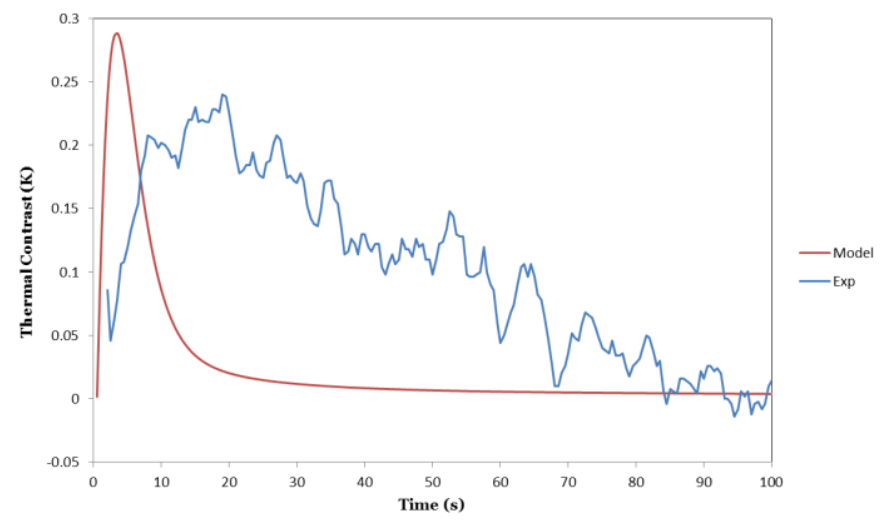

Fig. 20 Modelled and experimental surface thermal contrast data between defect and non-defect regions

\subsection{Pulse phase thermography results}

The real and imaginary components of the FFT have then been used to produce phase data for the DC and ND regions. Phase contrast values, $\Delta \phi$, have been produced over a range of sampling frequencies for both the experimental and modelled data, see Fig. 21a-d. The absence of noise in the experimental phase data is because the processed data is presented as the mean thermal decay for the defect and non-defect areas, as indicated in Fig. 12. The FFT sampled frequency at which the maximum $\Delta \phi$ occurs and the magnitude of this peak are recorded and the ratio between the modelled and experimental results was found, see Table 2. The magnitude of $\Delta \phi$ is consistently under predicted by the model with the magnitude of the peak being much larger in the experimental data. The FFT sweeps for a particular frequency component in the thermal decay signal for each pixel through time. As the FFT in PPT is identifying the frequency components of the thermal decay signal from the PT, any differences between the modelled and experimental PT data will be amplified by the algorithm that provides the phase data. The FFT has been carried out using

$$
F_{n}=\sum_{k=0}^{N-1} T(k) e^{2 \pi i k n / N}=\operatorname{Re}_{n}+i \operatorname{Im}_{n}
$$

where $n$ is the frequency increment, $T(k)$ is the temperature at a pixel for thermal image $k$ and $N$ is the total number of thermal images processed.

The phase data is calculated using

$$
\phi_{n}=\arctan \left(\frac{\operatorname{Im}_{n}}{\operatorname{Re}_{n}}\right)
$$


Equation (5) shows that the arctan function of $\mathrm{Im} / \mathrm{Re}$ calculates the phase which provides the amplification in contrast seen in Fig 7 that reveals the defect in the lap joint. However in devising a model for PPT it should be noted that the arctan operation will also magnify any difference caused by assumed material properties etc.

The FFT frequency at which the maximum $\Delta \phi$ occurs is accurately predicted for both the lap joint and concrete case studies. The frequency at which the peak phase contrast occurs in the aluminium case study is slightly over predicted at $1.111 \mathrm{~Hz}$ in the model compared to the experimental result of $0.871 \mathrm{~Hz}$. For the CFRP-PTFE case study the modelled frequency for the peak phase contrast is found at three times the experimental frequency of $0.281 \mathrm{~Hz}$ compared to the experimental value of $0.091 \mathrm{~Hz}$. The ability to be able to predict the frequency at which the peak will occur may allow reduced data processing as the range of frequencies required to be processed will be decreased. These results show that the data processing routine used shows promise, however, improvements are required in the thermal model to allow the $\Delta \phi$ magnitude data to be accurately predicted as currently differences between the thermal datasets are emphasised by processing into phase data.

a

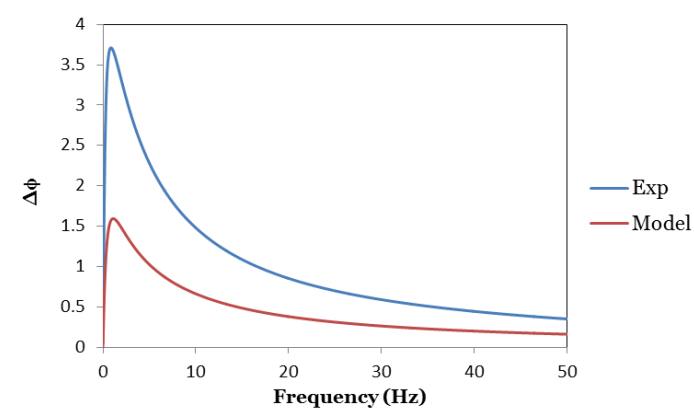

c

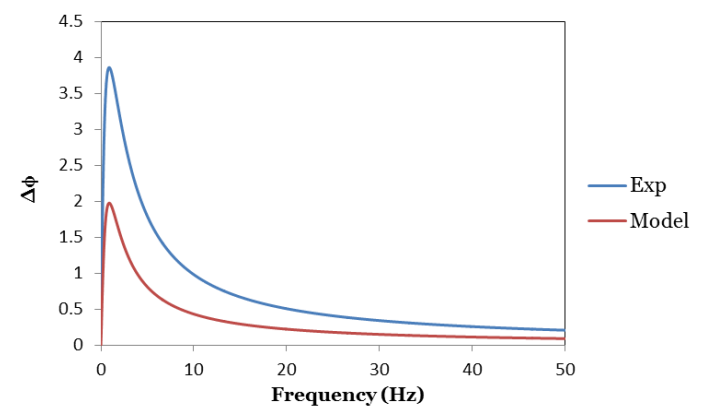

b

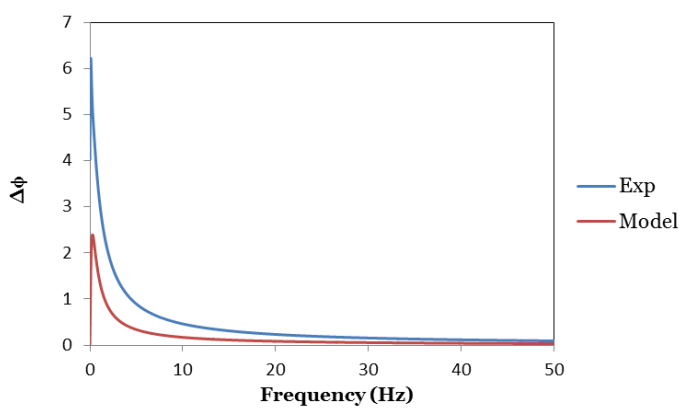

d

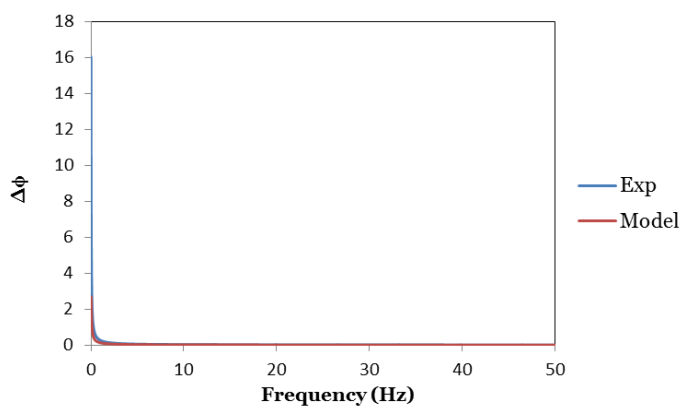

Fig. 21 Phase contrast between DC and ND for experimental and modelled data for a) CS1: Aluminium, b) CS2: CFRP with PTFE, c) CS3: CFRP-CFRP adhesive lap and d) CS4: CFRP-Concrete joint. 
Table 2 Ratio of modelled and experimental frequency at which the peak phase contrast occurs and ratio of magnitude of maximum phase contrast peak for each case study.

\begin{tabular}{|c|c|c|}
\hline Case study & $\begin{array}{c}\text { Frequency } \\
\text { Ratio }\end{array}$ & $\begin{array}{c}\text { Phase Contrast } \\
\text { Ratio }\end{array}$ \\
\hline Aluminium & 1.28 & 0.43 \\
\hline CFRP & 3.08 & 0.38 \\
\hline CFRP-CFRP Lap & 1.00 & 0.51 \\
\hline CFRP-Concrete & 1.00 & 0.17 \\
\hline
\end{tabular}

\section{FEA defect visibility study}

PPT relies on an underlying detectable thermal contrast of surface temperatures over defective and non-defective regions. Where this contrast is low the detectability of defects is greatly reduced. The previous case studies highlight examples where there is a strong contrast in thermal properties between defect and bulk material properties, such as CS1 and 2, and weak contrast, as in CS3. The current section of the paper aims to identify relationships between bulk and defect heat transfer properties relating to the detectability of the defect. Three common bulk materials have been investigated; aluminium, CFRP and glass fibre reinforced plastic (GFRP). The geometry of all of these samples has been set to $1 \mathrm{~mm}$ thick and $100 \mathrm{~mm}$ long with a $0.2 \mathrm{~mm}$ thick $10 \mathrm{~mm}$ long defect set at a depth of $0.4 \mathrm{~mm}$.

The effect of variation of the defect material diffusivity, relative to that of the bulk material, on the temperature contrast produced between defective and non-defective regions was investigated. Fig. 22a shows the ratio of defect to bulk material diffusivity plotted against the temperature contrast produced, $\Delta \mathrm{T}$. There is no temperature contrast when the defect and bulk material properties are the same, i.e. $\gamma_{D} / \gamma_{0}=1$. As the difference between defect and bulk properties is increased then $|\Delta \mathrm{T}|$ also increases, which enables defect identification. Where the defect has a higher diffusivity than the bulk material, $\gamma_{\mathrm{D}} / \gamma_{0}>1, \Delta \mathrm{T}$ is negative as the heat is transferred through the defect region, away from the surface, quicker than the surrounding defect free regions, resulting in a cool spot on the surface over the defect. When $\gamma_{\mathrm{D}} / \gamma_{0}<1, \Delta \mathrm{T}$ is positive as when a defect has a lower thermal diffusivity it hinders the propagation of heat causing an increased amount of reflection from the defect boundary which results in a hotspot on the surface over the defect. The heat must then either propagate through the defect of lower diffusivity or laterally around the defect, both of which will result in a distinctive perturbation in propagation of heat through the component. It is noted that defects with a diffusivity ratio less than 1 produce a much greater absolute temperature 
contrast to defect free material than those where the ratio is greater than 1 due to the variation in heat propagation. Fig. 22a shows that in the region surrounding $\gamma_{\mathrm{D}} / \gamma_{0}=1$, the $|\Delta \mathrm{T}|$ produced is insufficient to be reliably identified by the detector which has a thermal resolution of $20 \mathrm{mK}$. Within these regions there is no visible contrast using PT between the defective and defect free regions of the material. The size of these regions is proportional to the diffusivity of the bulk material. Threshold values of the diffusivity ratio outside of which the detection of defects is possible using PT are suggested in Table 3.

The modelled $\Delta \mathrm{T}$ data has then been processed into PPT $\Delta \phi$ data to show how the visibility of defects may be affected when presented as phase data, shown in Fig. 22 b. The magnitude of the $\Delta \phi$ data shows a similar asymmetric relationship as the original $\Delta \mathrm{T}$ data where a defect of lower diffusivity than the surrounding bulk material remains easier to identify than one of higher diffusivity. For all three materials processing the data into $\Delta \phi$ has the greatest effect on the visibility of defects with lower diffusivity than the bulk material while having a relatively small effect on defects of higher diffusivity. The visibility of the defects remains clearest in the aluminium however, the CFRP and GFRP show much closer results in terms of magnitude of $\Delta \phi$. The FFT approach used for producing the phase data has had the greatest effect on the defect visibility in the GFRP, i.e. the material with the lowest diffusivity. This is clearly a beneficial effect of the signal processing, as the FFT is applied over a range of frequencies so that an optimum frequency might be identified for a defect at a particular depth in a material. In Figure $22 \mathrm{~b}$ the maximum $\Delta \phi$ for has been plotted, hence accounting for the great improvement in identifying defects in the GFRP when using PPT instead of PT. This demonstrates that when $\Delta \mathrm{T}$ values are low it is most crucial that the FFT operates over the correct frequency range for the particular material.

The key feature of the data shown in Fig. $22 \mathrm{~b}$ is the effect of the phase processing on the extent of the central plateau region around $\gamma_{\mathrm{D}} / \gamma_{0}=1$. It is shown in Table 3 that while the extent of the $\Delta \phi$ plateau in the aluminium remains similar to that found in the $\Delta \mathrm{T}$ data it is shown that the plateaus for the composite materials of lower diffusivity are reduced. The reduction in the extent of the plateau regions enables defects of reduced contrast in diffusivity compared to the bulk material to become identifiable 
using PPT. Defects with diffusivity ratios within the remaining plateaus will remain undetected using PPT.

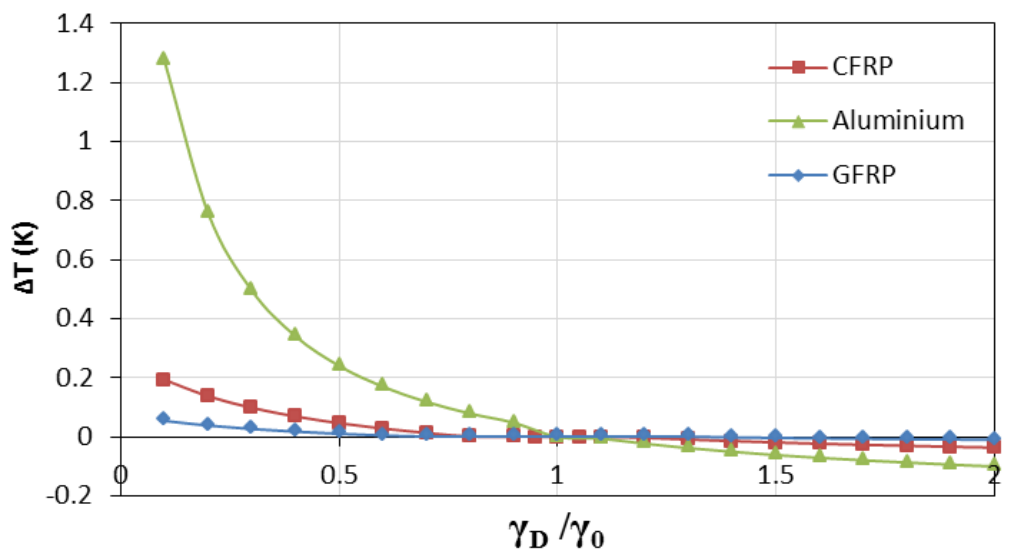

b

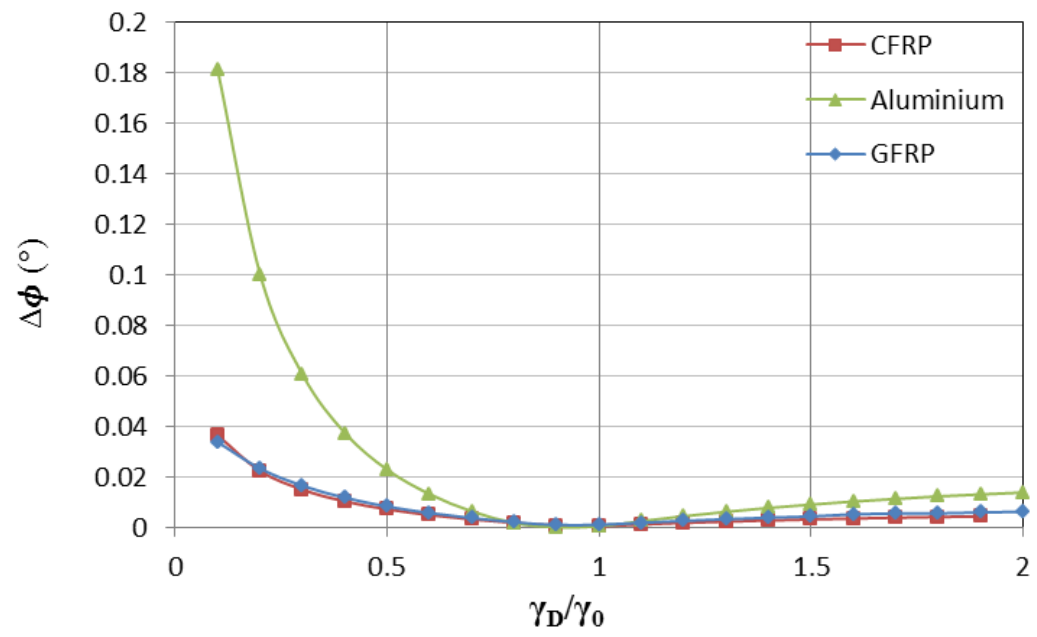

Fig. 22 a) $\Delta T$ values and b) $\Delta \phi$ values as ratio between defect and bulk thermal diffusivity, $\gamma_{\mathrm{D}} / \gamma_{0}$, is varied.

Table 3 Threshold values within which defects are no longer visible using PT $\Delta$ T values and PPT $\Delta \phi$ values as a percentage of bulk material property.

\begin{tabular}{|c|c|c|}
\hline Material & $\begin{array}{c}\gamma_{\mathbf{D}} / \gamma_{\mathbf{0}} \text { threshold } \\
\text { for PT }\end{array}$ & $\begin{array}{c}\gamma_{\mathbf{D}} / \gamma_{\mathbf{0}} \text { threshold } \\
\text { for PPT }\end{array}$ \\
\hline Aluminium & $< \pm 0.1$ & \pm 0.1 \\
\hline CFRP & \pm 0.2 & \pm 0.1 \\
\hline GFRP & \pm 0.3 & \pm 0.1 \\
\hline
\end{tabular}

It is noted that the four case studies presented in this paper have a diffusivity ratio of defect to immediately surrounding material of less than 1 and so are all visible. The geometry and presence of any additional materials of the samples has not been taken into account in this calculation of ratios. It is identified that if sufficient thermal excitation is applied to the components that the current defect materials have sufficient contrast of thermal diffusivity compared to the immediately surrounding material, i.e. adhesive for CS3 and CS4, for defects to be detectable. The overall detectability of 
defects in a component will also be dependent on the geometries of the surrounding material and the thermal pulse applied.

Another quantity that has been used in studies of defect visibility is 'effusivity', e.g. [19, 22]. Effusivity is the measure of the ease with which heat is able to transfer between two materials and is given by [23]

$$
e=\sqrt{k \rho C_{p}}
$$

Clearly this is important as the thermal contrast detected in thermography must be related to the heat transfer between one media and another. However, the model that has been developed in the present paper does not include the effect of effusivity but is based entirely on the Fourier diffusion relationship given by equation (1). A further point to note is the very good agreement between the experimental and modelled data for the pulsed thermography. Notwithstanding this, for completeness a study of the thermal and phase contrast in terms of relative effusivities was carried out and is shown in Figure 23 with threshold values also presented in Table 4. The trends are almost identical to those given in Figure 22 for the thermal contrast but with larger values of contrast. For the phase contrast, comparing effusivities provides different trends to the diffusivity comparison with more contrast. It is the square root in equation (6), which drives the more optimistic contrast values whereas in equation (2) there is a linear relationship between the material properties. The purpose of this section of work is to provide a means of determining if a specific defect type will be detectable. Hence comparing diffusivities provides the most conservative prediction as to whether a defect is detectable. The diffusion model has been validated with experimental data and is therefore considered to be reliable. 


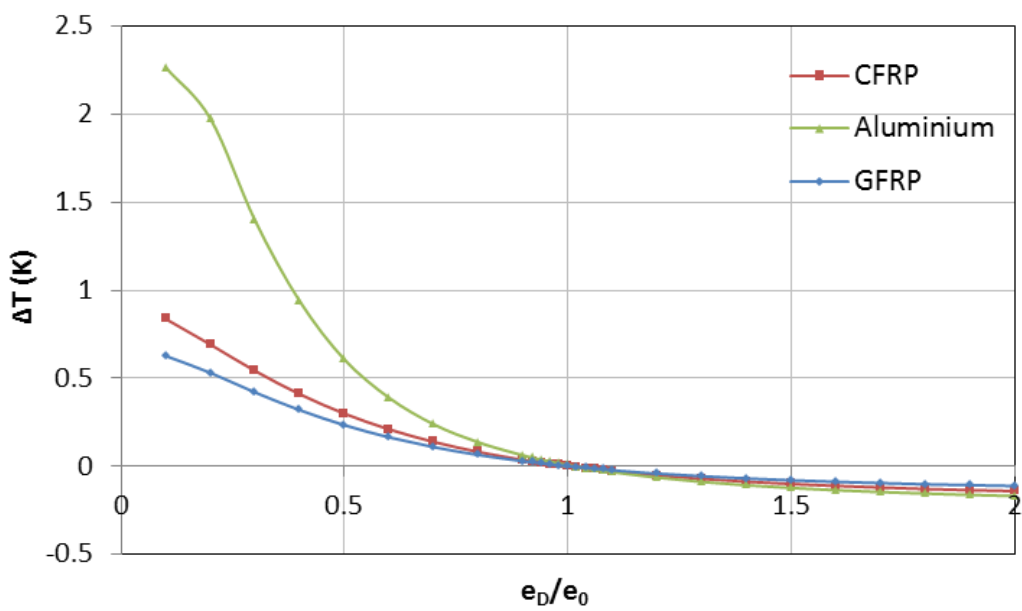

$\mathrm{b}$

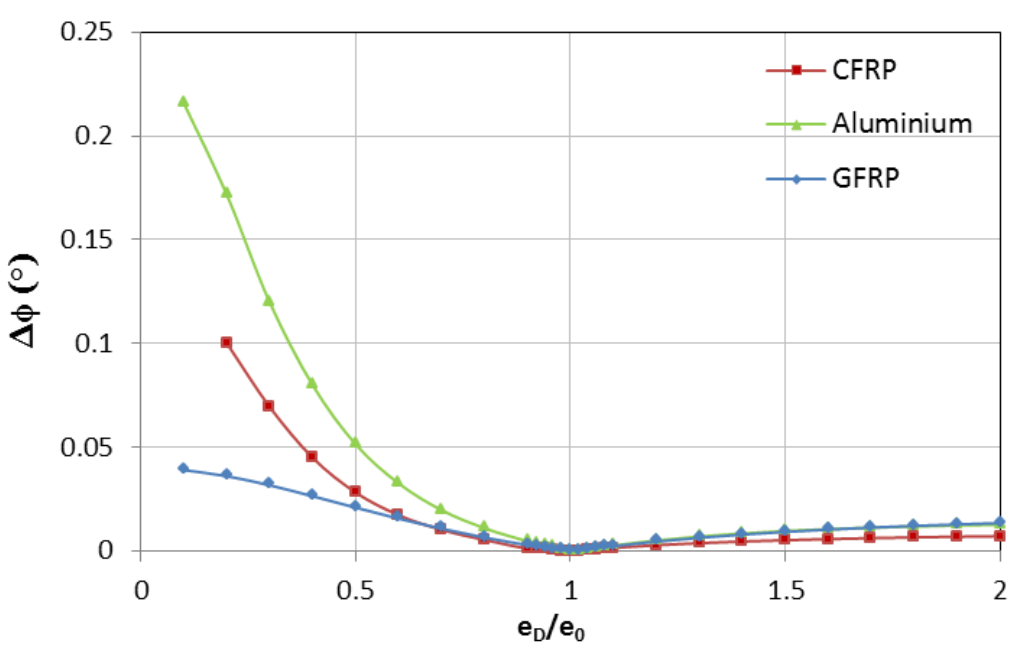

Fig. 23 a) $\Delta T$ values and b) $\Delta \phi$ values as ratio between defect and bulk thermal effusivity, $\mathrm{e}_{\mathrm{D}} / \mathrm{e}_{0}$, is varied.

Table 4 Threshold values within which defects are no longer visible using PT $\Delta \mathrm{T}$ values and PPT $\Delta \phi$ values with relation to thermal effusivity ratios.

\begin{tabular}{|c|c|c|c|c|}
\hline \multirow{2}{*}{ Material } & \multicolumn{2}{|c|}{$\mathbf{e}_{\mathbf{D}} / \mathbf{e}_{\mathbf{0}}$ threshold for PT } & \multicolumn{2}{c|}{$\begin{array}{c}\mathbf{e}_{\mathbf{D}} / \mathbf{e}_{\mathbf{0}} \text { threshold for } \\
\text { PPT }\end{array}$} \\
\cline { 2 - 5 } & Min limit & Max limit & Min limit & Max limit \\
\hline Aluminium & 0.98 & 1.06 & 0.98 & 1.02 \\
\hline CFRP & 0.96 & 1.06 & 0.98 & 1.04 \\
\hline GFRP & 0.94 & 1.08 & 0.98 & 1.02 \\
\hline
\end{tabular}

\section{Conclusions}

Four case studies (CS) were used to compare UT, PT and PPT experimental data and to compare the thermal experimental PT data with the modelled PT results. CS1 was an aluminium sheet with a flat bottom defect milled into the rear of the sample. CS2 was a CFRP panel with a PTFE defect added between plies during manufacture. CS3 was a CFRP single lap joint made using a standard epoxy adhesive with a PTFE defect 
introduced at the adhesive/adherend interface. Finally, CS4 was a CFRP patch adhered to a concrete structure with PTFE defect encapsulated in the adhesive layer.

The experimental PT thermal data, PPT phase data and UT C-scan image has been presented for the case studies. For CS1 and 2 the PT and PPT data was found to be comparable however CS3 and 4 demonstrate the advantages of PPT over PT. In CS3 the defect was not visible in the PT data whereas in the PPT phase image the defect was clearly visible. The PT data presented for CS4 does reveal the defect of interest but there was some reflection on the surface which may detract from defect identification. The reflections are greatly reduced in the PPT phase image, enabling more reliable defect identification. The UT data consistently revealed the defects in each case, however, the scans taken were always far more time consuming than the thermography methods. The fact that UT scanning is a point by point process is the main drawback of UT techniques.

The model and PT data comparison focussed on three key points on the surface above the defect centre (DC), defect edge (DE) and a non-defect area (ND) and the thermal contrast between DC and ND. The data for corresponding points in the modelled and PT data was taken for comparison. In CS1 the data compared well although an additional effect was revealed in the model, the inversion of the decay curves which was not identified in the PT data. Whilst this may be present in the experimental decay it was found that this effect it would be likely to be hidden by experimental noise. In CS2 and 3 the modelled and experimental data compared very well, however there was more of a discrepancy in CS4 where the spread of the decay curves was significantly reduced in the model data from $0.3 \mathrm{~K}$ in the experimental to $0.07 \mathrm{~K}$ in the model. The main difference between CS4 and the other studies was the ability to obtain reliable material properties for the specific materials in components. Whereas CS3 used a common epoxy adhesive to make the lap joint the adhesive used to join the concrete and CFRP in CS4 was much less common and so the thermal material properties were less well documented. It is believed the less reliable properties used in the case of CS4 has led to the errors found in the spread of the decay curves although their relative positions are correct. The thermal contrast data for CS2 and 3 demonstrating that the model is capturing the thermal contrast evolution produced in the experiments. The model of CS4 is not able to predict the thermal contrast evolution. 
PPT phase contrast data has been presented for the modelled and experimental results. The processing routine used under predicted the magnitude of the phase contrast but had some success the frequency at which the peak $\Delta \phi$ occurred. The discrepancies are caused by the inverse tan processing involved in the production of the phase data as this emphasises small differences in data, which is why the phase data reveals defects that were not visible in the thermal data. Overall the advantages of the PPT phase data over the PT and UT data has been highlighted and model designed to be comparable to PT has performed well where accurate material properties are known.

A defect detectability study was carried out. It was found that the presence of $\Delta \mathrm{T}$ and $\Delta \phi$ regions surrounding $\gamma_{\mathrm{D}} / \gamma_{0}=1$ where defects are not identifiable may be related to defect detectability and threshold values where defects are able to be identified have been suggested. Generally processing of the PT thermal data into PPT phase data has been found to reduce the extent of plateau regions surrounding $\gamma_{\mathrm{D}} / \gamma_{0}=1$ and hence enhance detectability of defects with lower thermal contrast. Neither PT nor PPT is able to detect kissing defects that would occur within the region around $\gamma_{\mathrm{D}} / \gamma_{0}=1$. The application of a small amount of load may be sufficient to enhance defect detectability and enable the detection of kissing defects using PT or PPT. This is the subject of a future paper [15]. Future work will also include predictions of defect depth using the approaches described in the paper.

\section{Acknowledgements}

The Lloyd's Register Foundation and the EPSRC are acknowledged for the continued financial support of this work.

\section{References}

1. Baker, A., Bonded composite repair of fatigue-cracked primary aircraft structure. Composite Structures, 1999. 47: p. 431-443.

2. Adams, R.D. and Cawley, P., A review of defect types and nondestructive testing techniques for composites and bonded joints. NDT International, 1988. 21(4): p. 208-222.

3. Marty, P.N., Desai, N., and Andersson, J. NDT of kissing bond in aeronautical structures. in $16^{\text {th }}$ World Conference on NDT. 2004. Montreal, Canada.

4. Jeenjitkaew, C., Luklinska, Z., and Guild, F., Morphology and surface chemistry of kissing bonds in adhesive joints produced by surface contamination. International Journal of Adhesion \& Adhesives, 2010. 30: p. 643-653.

5. Polimeno, U., Meo, M., Almond, D.P., and Angioni, S.L., Detecting low velocity impact damage in composite plate using nonlinear acoustic/ultrasound methods. Applied Composite Materials, 2010. 17: p. 481-488.

6. Schilling, P.J., Karedla, B.P.R., Tatiparthi, A.K., and Verges, M.A., X-ray computed microtomography of internal damage in fiber reinforced polmer matrix composites. Composites Science and Technology, 2005. 65. 
7. Hung, Y.Y., Shearography for Non-destructive Evaluation of Composite Structures. Optics and Lasers in Engineering, 1996. 24: p. 161-182.

8. De Angelis, G., Meo, M., Almond, D.P., Pickering, S.G., and Angioni, S.L., A new technique to detect defect size and depth in composite structure using digital shearography and unconstrained optimization. NDT\&E International, 2012. 45(1): p. 91-96.

9. Avdelidis, N.P., Almond, D.P., Dobbinson, A., and Hawtin, B.C., Aircraft composites assessment by means of transient thermal NDT. Progress in Aerospace Sciences, 2004. 40: p. 143-162.

10. Brotherhood, C.J., Drinkwater, B.W., and Dixon, S., The detectability of kissing bonds in adhesive joints using ultrasonic techniques. Ultrasonics, 2003. 41: p. 521-529.

11. Yan, D., Drinkwater, B.W., and Neild, S.A. Experimental and theoretical characterisation of kissing bonds in adhesive joint using non-linear ultrasonic measurement. in AIP Conference Proceedings. 2010.

12. Hung, M.Y.Y., Chen, Y.S., and Ng, S.P., Review and comparison of shearography and pulsed thermography for adhesive bond evaluation. Optical Engineering, 2007. 46(5): p. 051007.1 051007.16

13. Junyan, L., Liqiang, L., and Yang, W., Experimental study on active infrared thermography as a NDI tool for carbon-carbon composites. Composites: Part B, 2013. 45: p. 138-147.

14. Manohar, A. and Lanza di Scalea, F., Determination of defect depth and size using virtual heat sources in pulsed infrared thermography. Experimental Mechanics, 2013. 53: p. 661-671.

15. Waugh, R.C., Dulieu-Barton, J.M. and Quinn, S., Infrared thermography to identify low volume defects in adhesive bonds. Submitted 2013.

16. Yan, D., Neild, S.A., and Drinkwater, B.W., Modelling and measurement of the nonlinear behaviour of kissing bonds in adhesive joints. NDT\&E International, 2012. 47: p. 18-25.

17. Maldague, X., Introduction to NDT by active infrared thermography, Materials Evaluation, 2002. 6(9): p. 1060-1073.

18. Sun, J.G., Analysis of pulsed thermography methods for defect depth prediction. Journal of Heat Transfer, 2006. 128: p. 329-338.

19. Maldague, X., Theory and practice of infrared technology for nondestructive testing. Wiley series in microwave and optical engineering, K. Chang 2001, Chichester: John Wiley \& Sons, Inc.

20. Maldague, X. and Marinetti, S., Pulse phase infrared thermography. Journal of Applied Physics, 1996. 79(5): p. $2694-2698$.

21. www.matweb.com, MatWeb - Material property data. [cited 6th May 2012]

22. Ibarra-Castanedo, I. and Maldague, X., Pulsed phase thermography reviewed. Quantitative Infrared Thermography Journal, 2004. 1:1, p. 47 - 70.

23. Kaviany, M., Essentials of heat transfer. Cambridge University Press, New York, 2011. 